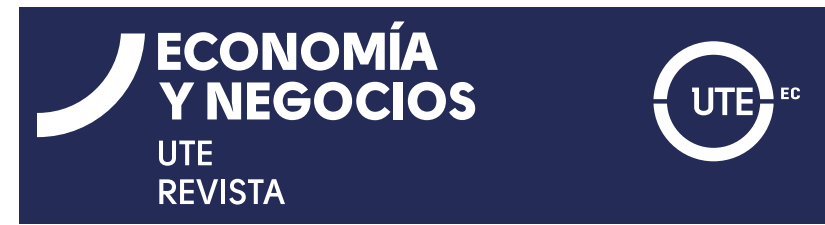

Economía y Negocios, 2021, 12(02), 18-31.

http://revistas.ute.edu.ec/index.php/economia-y-negocios

\section{ARTÍCULO DE INVESTIGACIÓN}

ISSN-e: 2602-8050

Recibido (Received): 2021/09/05 Aceptado (Accepted): 2021/10/15

Publicado (Published): 2021/12/06

https://doi.org/10.29019/eyn.v12i2.944

\title{
Variables Determinantes de las Prácticas de Gestión de Recursos Humanos en Micro y Pequeñas Empresas
}

\section{Determinant Variables of Human Resources Management Practices in Micro and Small Businesses}

\author{
Reyner PÉREZ-CAMPDESUÑER' (D), Roberto MARRERO-ARIAS ${ }^{2}$ (D) y Margarita de MIGUEL-GUZMÁN ${ }^{3}$ (D) \\ 1. Universidad UTE, Facultad de Ciencias Administrativas. Santo Domingo, Ecuador. \\ 2. Universidad de Holguín, Facultad de Ingeniería Industrial. Holguín, Cuba. \\ 3. Instituto Superior Tecnológico Atlantic, Departamento de Administración. Santo Domingo, Ecuador.
}

Email: reyner.perez@ute.edu.ec; robertomcu@uho.edu.cu; maguyaefdpdm@gmail.com

\section{Resumen}

En la actualidad, son escasas las investigaciones que profundizan en los pormenores de la gestión del talento humano para micro y pequeñas empresas. Por medio de esta investigación se pretende caracterizar en qué medida las prácticas de gestión de recursos humanos (GRH) de las micro y pequeñas empresas en Ecuador difieren o se asemejan a las prácticas de esta disciplina en las medianas y grandes empresas.

Para este fin se tuvo acceso a información sobre la forma en que se desarrolla esta área de gestión en 974 organizaciones representativas de los distintos estratos del universo de empresas de la población en estudio, y sobre el grado de aplicación de las distintas funciones. Además, se evaluaron otras variables como el número de trabajadores y la edad de la empresa.

Como resultado fundamental se estableció la existencia de niveles de correlación entre el grado de desarrollo de la actividad y el tamaño y antigüedad de la empresa. Asimismo, se encontró que el nivel de desarrollo de las funciones de GRH, en particular, y de la actividad, en general, varía en correspondencia con el tipo de organización.

\section{Palabras clave}

Gestión de recursos humanos, micro y pequeñas empresas, empresas medianas y grandes.

\section{Abstract}

Currently, there is little research that delves into the details of human talent management for micro and small companies

Through this research, the aim is to characterize the extent to which human resource management (HRM) practices of micro and small companies in Ecuador differ or resemble the practices of this discipline in medium and large companies. For this purpose, access to information was had on the way in which this management area is developed in 974 representative organizations of the different strata of the universe of companies of the population under study, and on the degree of application of the different functions. In addition, other variables such as the number of workers and the age of the company were evaluated.

As a fundamental result, the existence of levels of correlation between the degree of development of the activity and the size and age of the company was established. Likewise, it was found that the level of development of HRM functions, in particular, and of the activity, in general, varies in correspondence with the type of organization.

\section{Keywords}

Human resources management, micro and small enterprises, medium and large enterprises.

Códigos de clasificación JEL: M10, M12, L26. 


\section{Introducción}

De acuerdo con Krishnan y Hug (2017), las micro, pequeñas y medianas empresas (mipymes) ocupan un lugar predominante en lo relativo al porcentaje del total de empresas en la mayoría de los países. En los EE.UU., cerca del $99 \%$ de las empresas comerciales son mipymes y representan el $52 \%$ del empleo total, y en la Unión Europea cerca del $66 \%$ de los puestos de trabajo lo aportan las mipymes. Asimismo, las mipymes forman el núcleo de la economía de la región de AsiaPacífico, es decir, el 30 y el $50 \%$ del empleo proviene de este sector. En el informe de Organización para la Cooperación y el Desarrollo Económicos (OECD, 2015) se señaló que más del 99 \% de las empresas de los países de la organización y del G20 son mipymes.

Los valores que se utilizan para la clasificación en mipymes varían de un país a otro. Mientras que en EE.UU. pueden llegar a tener hasta 500 trabajadores y en Europa a 250, en Ecuador puede tener hasta $49 \mathrm{em}$ pleados. En Ecuador, además, se clasifican estas en función del volumen de ventas que generan, lo que puede resultar confuso dado que pueden cumplir un criterio, pero otro no.

A pesar de lo representativas que resultan las mipymes, Barrett (2006, p. 445), citando a Tansky y Heneman, dice: "las pequeñas y medianas empresas han sido tratadas como ciudadanas de segunda clase por los autores en la literatura sobre gestión de recursos humanos durante demasiado tiempo". Si bien esta situación ha mejorado porque cada día se profundiza y aporta a este objeto de estudio, se puede afirmar que aún es insuficiente. No todos reconocen que las herramientas y métodos diseñados para las grandes empresas no funcionan de igual modo en las condiciones de las mipymes que, por su naturaleza, son distintas.

Daspit et al. (2018) reconoce la importancia del talento humano cuando enuncia su potencial para generar la ventaja competitiva de la empresa. Según Krishnan y Hug (2017), la atracción, motivación, desarrollo y retención de los trabajadores son reconocidas desde hace mucho tiempo como un reto importante al que se enfrentan las mipymes. Por esta razón, en los últimos años se reporta un crecimiento de la investigación sobre los temas de GRH en el contexto de las mipymes. El mismo autor considera que de forma frecuente las prácticas de las grandes empresas se aplican de forma acrítica en las mipymes, sin tomar en cuenta las particularidades de estas organizaciones (Ahammad et al., 2020)

El objeto de esta investigación es caracterizar en qué medida las prácticas de GRH de las mipymes en Ecuador difieren o se asemejan a las prácticas de esta disciplina en las medianas y grandes empresas.

\section{Revisión de la literatura}

Se revisaron los artículos publicados en dos de las revistas que mejor indexación presentan sobre el tema: Journal of Human Resources y Human Resource Management Review, ambas indexadas en Scopus, con un SJR de 4.77 y 2.55 respectivamente, lo cual permitió identificar la existencia de 1770 artículos relacionados con la GRH. De estos, solo se consideraron 1353, el resto estaban integrados por cartas del editor, introducciones, resúmenes de números de las revistas o aclaraciones sobre artículos ya publicados.

Con base en los artículos consultados, y aunque es reconocido el carácter sistémico de la GRH, quienes investigan han profundizado en uno de los subsistemas o funciones que intervienen en el desarrollo de esta. En consecuencia, se debe destacar que, si bien todas las funciones son analizadas, no lo son con la misma frecuencia y profundidad.

En la figura 1 se muestra un resumen de cómo es abordada cada una de las funciones, además se muestra las investiga- 
ciones en las que se ha profundizado en la concepción del sistema, la influencia del entorno, así como los principales resultados que se vinculan con la GRH. Como se puede ver, aunque se analizan cada una de las fun- ciones, las más comunes son la recompensa, la formación y el reclutamiento y selección (Acikgoz, 2019; Ahammad et al., 2020; Johnson \& Roberto, 2019).

Figura 1. Comportamiento de las publicaciones sobre GRH

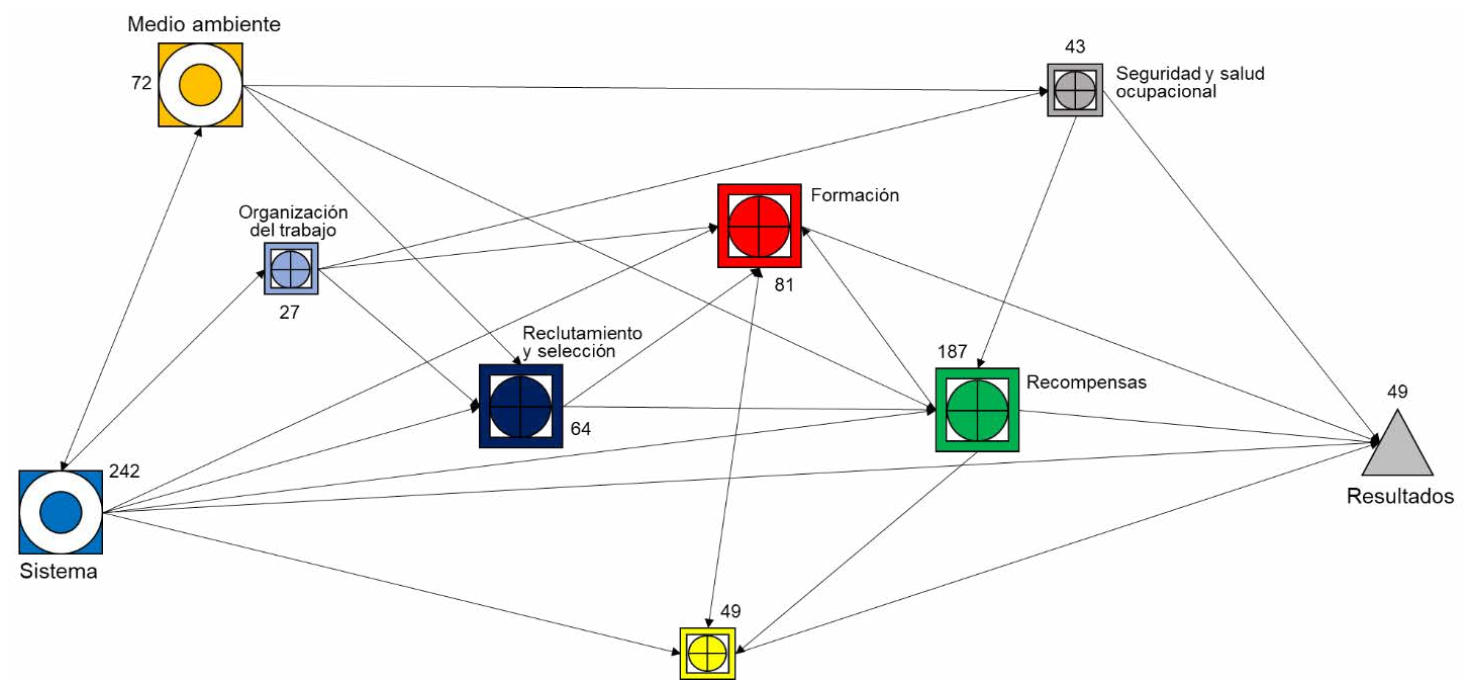

Krishnan y Hug (2017), basándose en varios autores, expone la idea de que las prácticas de recursos humanos varían según el tamaño de la organización. Mientras más grande es la organización, mayor la complejidad de las funciones de GRH que esta puede asumir. En cambio, las mipymes, al disponer generalmente de menos recursos, gastan menos en la actividad y le otorgan un carácter más operativo. El autor, además, considera como otra fuente de diferencias el sector en que se desarrollan las organizaciones y plantea que las causas de las diferencias se pueden explicar a partir de tres teorías:

- La teoría institucional: las organizaciones son entidades sociales que buscan la aprobación de su desempeño en una estructura socialmente construida.

- La teoría económica: las economías de escala deben ser alcanzadas antes de que se puedan implementar prácticas sofisticadas de recursos humanos.
- La teoría de la dependencia de recursos: todas las organizaciones dependen de recursos externos, críticos para su supervivencia, y esto, a su vez, influye en el comportamiento de la empresa.

Daspit et al. (2018) plantea que las organizaciones familiares representan cerca del 90 \% o más de todas las empresas, porque emplean al $50 \%$ de la población activa y crean el $75 \%$ de los nuevos puestos de trabajo en todo el mundo. Combs et al. (2018), explica que estas organizaciones difieren considerablemente de las empresas no familiares en sus objetivos, comportamientos y resultados, porque persiguen metas únicas, como el deseo de pasar la firma a la próxima generación, y sus objetivos únicos fomentan regularmente comportamientos distintivos. Por ejemplo, las empresas familiares son más propensas a contratar a miembros de la familia y, de modo general, más reticentes a despedir empleados.

Estas organizaciones, formula Daspit et al. (2018), tienen fama de mostrar favo- 
ritismo hacia los miembros de la familia en su selección de recursos humanos, en las prácticas salariales y en las acciones de formación, por lo que estas diferencias también generan comportamientos distintivos. Mientras que la productividad de los miembros de la familia puede verse disminuida, los miembros que no pertenecen a ella tienen más probabilidades de ser compensados en función del mérito en dicho sistema, lo que mejora la productividad (Daspit et al., 2018).

Krishnan y Hug (2017) explica que en las mipymes los empleados se seleccionan en función de lo bien que encajan en la cultura firme. Se presta mucha atención a los factores como la coincidencia de normas, valores y creencias que el solicitante debe compartir con la organización. Es probable que los efectos de una mala selección se amplifiquen en estas organizaciones. En la medida que una organización crece, los roles amplios superpuestos dan paso a roles más especializados y se adopta una estructura funcional. En igual dirección disminuye la polivalencia de sus empleados por dos razones: el incremento de la capacidad de especificar trabajos con mayor descripción formal y la necesidad de contar con especialistas en esos trabajos. Con el aumento del tamaño de la organización, los directivos se orientan a la concepción estratégica y delegan las responsabilidades tácticas.

Cardon y Stevens (2004) dicen que el reclutamiento y selección de personal es quizás el tema de recursos humanos más examinado en el contexto de las mipymes. Aunque generalmente estas empresas no tienen departamentos de recursos humanos, todas las organizaciones poseen políticas de contratación y recursos humanos, aun cuando estas no estén escritas y la mayoría de las funciones de GRH las asuma el gerente (Stone \& Stone, 2015).

Si bien la función de reclutamiento y selección es de las más utilizadas por las mi- pymes existen algunas dificultades respecto a este tipo de organizaciones, condicionadas por la escasez de recursos, la falta de reconocimiento del empleador y la ausencia de calificadores de cargos, lo cual demanda alta polivalencia; además, no es una función que se ejecute de forma sistemática. En este tipo de organizaciones la selección se basa principalmente en hacer entrevistas, evaluar la experiencia previa y los requisitos educativos para el trabajo, y verificar los antecedentes y las referencias. Asimismo, durante el proceso de selección, otorgan gran peso a las normas, valores y creencias de la organización y los solicitantes, y el grado de correspondencia de la persona-organización (Acikgoz, 2019; Johnson \& Roberto, 2019).

En correspondencia con lo que reflejan las investigaciones (Cardon \& Stevens, 2004; Krishnan \& Hug, 2017), la función de compensación es otra de las más abordadas en estas organizaciones. La mayor parte del trabajo en la compensación se orienta a la combinación salarial y en la generación de métodos para pagar incentivos. Los incentivos a corto plazo en varias ocasiones se relacionan con la participación en las ganancias y en las acciones, donde la estimulación solo se entrega cuando las ganancias a nivel organizacional son amplias. Por lo general, este tipo de organizaciones tiene una estructura salarial con pocos niveles de gestión y tienden a tratar a los empleados de forma igualitaria (Bush, 2020).

Respecto a la función de formación, se asume que esta no está estandarizada y que se deben desarrollar acciones de este tipo en correspondencia con el tipo de negocio. La acción de formación más fácil de identificar es el entrenamiento para nuevos puestos de trabajo. Otra acción de formación que se identifica es la del proceso de socialización que se lleva a cabo cuando se incorpora un nuevo miembro a la organización (Batistič, 2018). Además, por la propia 
naturaleza de la organización, se propicia una alta polivalencia para asumir los diferentes contenidos de trabajo.

Finalmente, Cardon y Stevens (2004) señalan que los estudios relacionados con la organización del trabajo y la evaluación del desempeño en las pequeñas y medianas empresas son escasos, lo cual deja una brecha para esta investigación (Wilkinson et al., 2020).

\section{Materiales y métodos}

Para este estudio se siguieron los siguientes pasos:

\section{Diseño de los instrumentos de investigación}

Para caracterizar el grado de desarrollo de la GRH en estas organizaciones, se definió un grupo de ítems para evaluar el grado de presencia de estos en las mipymes. Los ítems fueron establecidos en correspondencia con las funciones de la GRH y las prácticas habituales asociadas a estas, así como algunos requisitos que son una expresión del grado de desarrollo de la actividad. En la tabla 1 se muestra un resumen de cada uno de los ítems definidos por funciones.

Tabla 1. Conjunto de ítems por evaluar por función de la GRH

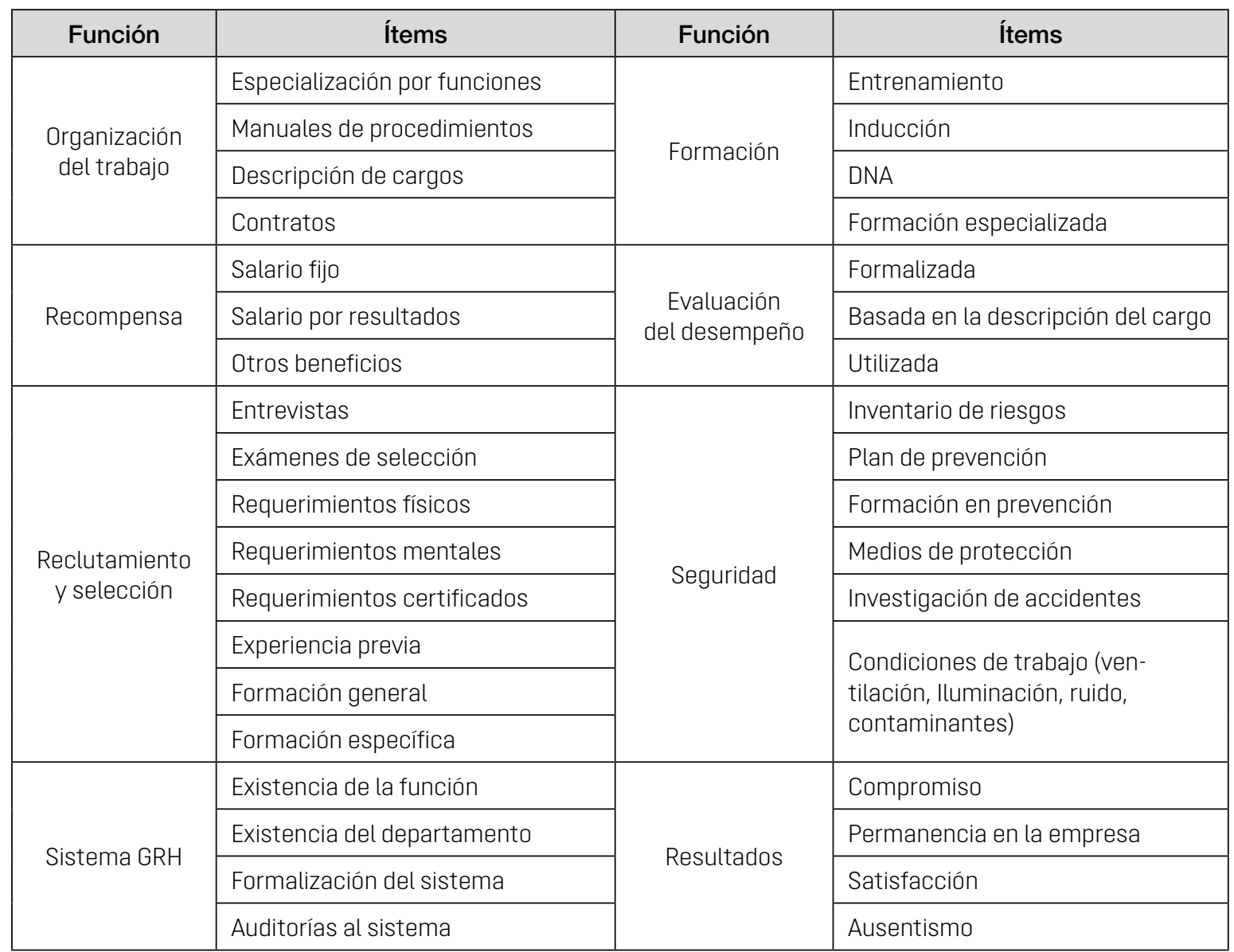

El grado de presencia de cada uno de estos ítems y variables fue evaluado mediante la aplicación de un cuestionario a cada una de las entidades seleccionadas para el estudio. 


\section{Definición de la población y la muestra}

De acuerdo con los datos del Instituto de Estadística y Censo de Ecuador (INEC, 2018), en el país se encontraban registradas 823005 em- presas distribuidas por tipos de empresas (tabla 2). Como se puede constatar, existe un alto predominio de las micro y pequeñas empresas.

Tabla 2. Composición de la población y la muestra de la investigación

\begin{tabular}{|l|c|c|c|c|}
\hline \multirow{2}{*}{ Tipo de empresas } & \multirow{2}{*}{ Población } & \multicolumn{2}{c|}{ Muestra } & \multirow{2}{*}{$\begin{array}{c}\text { Valor real } \\
\text { de } \text { e }^{*}\end{array}$} \\
\cline { 3 - 4 } & & Plan & Real & 0.05 \\
\hline Micro & 773772 & 384 & 379 & 0.05 \\
\hline Pequeñas & 41647 & 381 & 329 & 0.07 \\
\hline Medianas & 6344 & 363 & 192 & 0.10 \\
\hline Grandes & 1242 & 294 & 89 & \\
\hline Total & 823005 & 1422 & 974 & \\
\hline
\end{tabular}

Nota: *e es el valor real del error del investigador (e) (Ecuación 2).

Para definir el tamaño de la muestra, se consideró cada tipología de las empresas como poblaciones diferentes, dado que tie- nen características diferentes entre sí. La determinación del tamaño de la muestra se calculó con la ecuación 1.

$$
\text { Ecuación 1: } n=\frac{N^{*} p^{*} q^{*} z^{2}}{e^{2 *}(N-1)+z^{2 *} p^{*} q}
$$

Donde:

$\mathrm{N}$ : tamaño de la población

p: probabilidad de éxito $(0.5)$

q: probabilidad de fallo (0.5)

e: error del investigador (5\%)

z: constante de la distribución normal: 1.96 para el $95.5 \%$ de nivel de confianza.

No se logró acceder al total de la muestra prevista porque muchas empresas no permitieron acceder a la información, por lo que fue necesario calcular el valor real del error del investigador (e) a partir de la ecua- ción 2. Como se puede ver en la tabla 2, en todos los casos se garantiza un error del investigador, menor de $10 \%$, lo cual se considera admisible.

$$
\text { Ecuación 2: } \mathrm{e}=0.98 * \sqrt{\frac{N-n}{n^{*}(N-1)}}
$$

En la figura 2 se caracteriza la composición de la muestra general por sector de la economía. Existe un predominio del sector de los servicios y del comercio, lo que corresponde con la estructura general de las empresas en Ecuador y otros países (tabla 3). 
Tabla 3. Composición de la muestra por sector

\begin{tabular}{|l|c|c|c|c|c|c|}
\hline \multicolumn{1}{|c|}{ Sector productivo } & Micro & Pequeña & Mediana & Larga & Total & Porcentaje \\
\hline Agricultura & 4 & 32 & 22 & 11 & 69 & 7 \\
\hline Producción & 3 & 21 & 35 & 25 & 84 & 8 \\
\hline Comercio & 138 & 79 & 38 & 22 & 277 & 28 \\
\hline Construcción & 0 & 4 & 9 & 7 & 20 & 2 \\
\hline Servicios & 234 & 193 & 88 & 24 & 539 & 55 \\
\hline Total & 379 & 329 & 192 & 89 & 989 & 100 \\
\hline
\end{tabular}

\section{Diseño de la forma de procesamiento}

En cuanto a la información, se partió del análisis de la relación entre el nivel de desarrollo de la GRH y la cantidad de personas en la organización y, de igual forma, la relación con el tiempo de fundada la empresa (edad). Para este análisis se determinó el valor de desarrollo de la GRH a partir del grado de presencia o no de cada una de las variables evaluadas, para esto se utilizó la ecuación 3.

\section{Ecuación 3: $\mathrm{SGRH}=\sum_{i=1}^{n}$ Grado de presencia del item $i$ en la empresa}

Para analizar la relación entre las variables se utilizó el coeficiente de correlación de Pearson $\mathrm{R}^{2}$. Posteriormente, se caracterizó el grado de presencia de cada una de las funciones de GRH en las organizaciones y se evaluó si existen diferencias significativas de este, según el tipo de empresa o el sector al que esta pertenece. En el análisis de las diferencias significativas se aplicó la prueba de hipótesis para diferencias de medias en muestras independientes con el estadígrafo de Kruskal-Wallis. El grado de presencia de cada una de las funciones se determinó a partir del análisis del grado de presencia de los ítems evaluados para su caracterización.

\section{Análisis y resultados}

El análisis del nivel de correlación entre la cantidad de personas o la edad de la empresa con el grado de desarrollo de la actividad de GRH se resume en la tabla 4. En la tabla existen niveles de correlación entre la edad de las empresas y el grado de desarrollo de la GRH, dado que en todos los casos resultó muy significativo el coeficiente de correlación. Sin embargo, no se observó correlación entre la cantidad de trabajadores y el grado de desarrollo de la $\mathrm{GRH}$, independientemente del tipo de empresas que se analice. No obstante, sí se encontró correlación entre la cantidad de personas en general y el nivel de desarrollo de la GRH.

De acuerdo con los resultados de la figura 2, las microempresas crecen en su nivel de desarrollo de la GRH, pero no logran desarrollar esta función empresarial en todo su esplendor, lo que muestra un comportamiento asintótico cuando el grado de la GRH alcanza un valor medio, donde se considera como máximo la manifestación de todas las actividades analizadas. 
Tabla 4. Correlación entre cantidad de personas y edad y el grado de desarrollo de la GRH

\begin{tabular}{|c|l|l|}
\hline \multirow{2}{*}{ Tipo de empresas } & \multicolumn{2}{|c|}{ Variables } \\
\cline { 2 - 3 } & & \multicolumn{1}{|c|}{ GRH } \\
\hline \multirow{2}{*}{ Micro } & Personas & 0.035 \\
\cline { 2 - 3 } & Edad & $0.798^{\star *}$ \\
\hline \multirow{2}{*}{ Pequeñas } & Personas & 0.084 \\
\cline { 2 - 3 } & Edad & $0.933^{\star *}$ \\
\hline \multirow{2}{*}{ Medianas } & Personas & 0.04 \\
\cline { 2 - 3 } & Edad & $0.916^{\star *}$ \\
\hline \multirow{2}{*}{ Grandes } & Personas & 0.004 \\
\cline { 2 - 3 } & Edad & $0.732^{\star *}$ \\
\hline \multirow{2}{*}{ Total } & Personas & $0.674^{\star *}$ \\
\cline { 2 - 3 } & Edad & $0.582^{\star *}$ \\
\hline
\end{tabular}

Nota: ** altamente significativo.

Figura 2. Relación entre el desarrollo de la GRH y la cantidad de personas
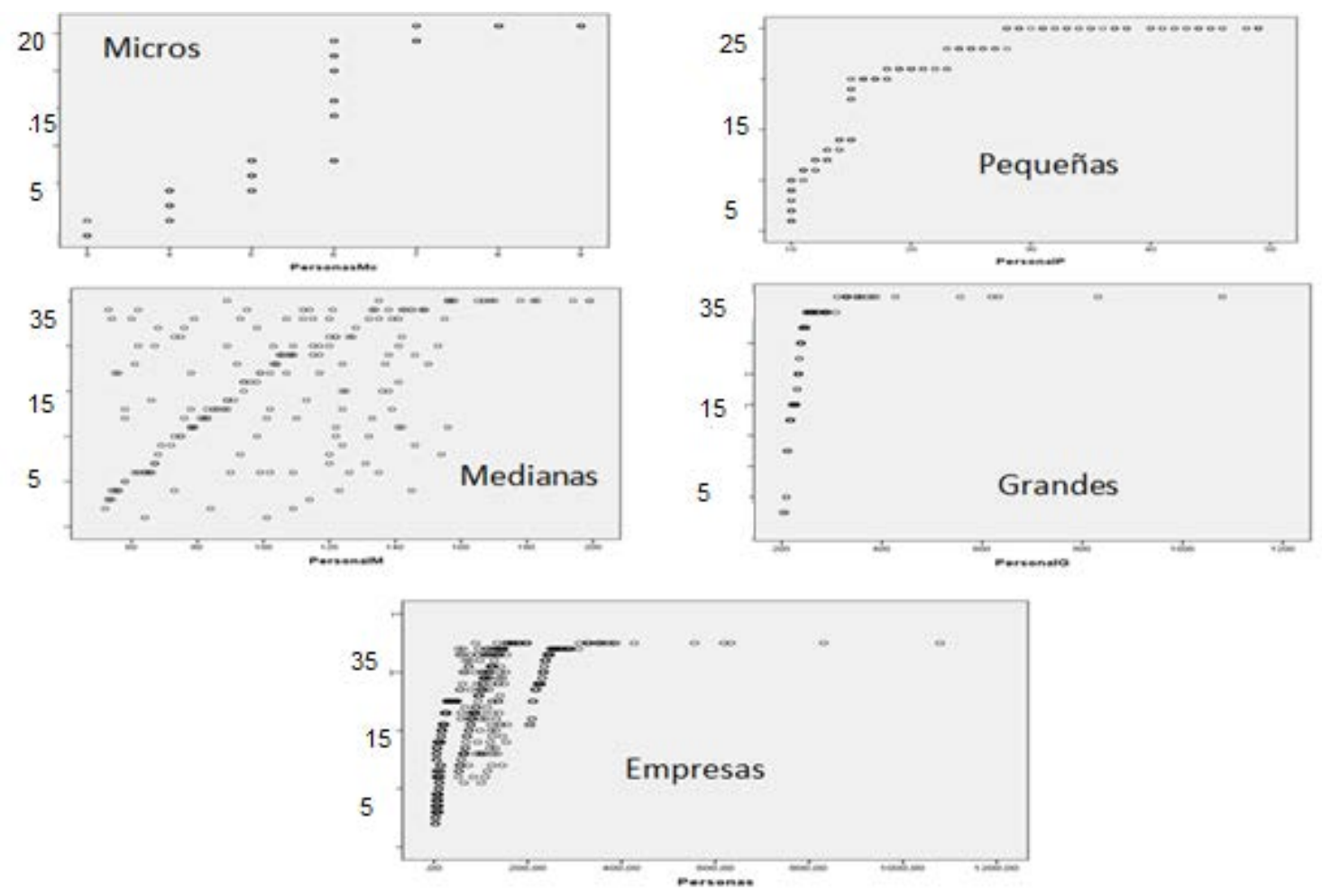

Las empresas pequeñas muestran un comportamiento similar, solo que el valor de la asintota se manifiesta con un valor mayor que en el caso de las microempresas.
Las empresas medianas y grandes tienden a mostrar un crecimiento más rápido en su nivel de desarrollo de la GRH y logran alcanzar el máximo desarrollo de esta, aunque se 
puede constatar que las empresas medianas muestran una mayor dispersión.

De igual modo, en la figura 3 se reflejan las relaciones entre la edad de las empresas y el grado de desarrollo de la GRH. En este caso, las relaciones son más explícitas en los gráficos, independientemente del tipo de empresas y con menor nivel de dispersión.

Figura 3. Relación entre el desarrollo de la GRH y la edad de las empresas
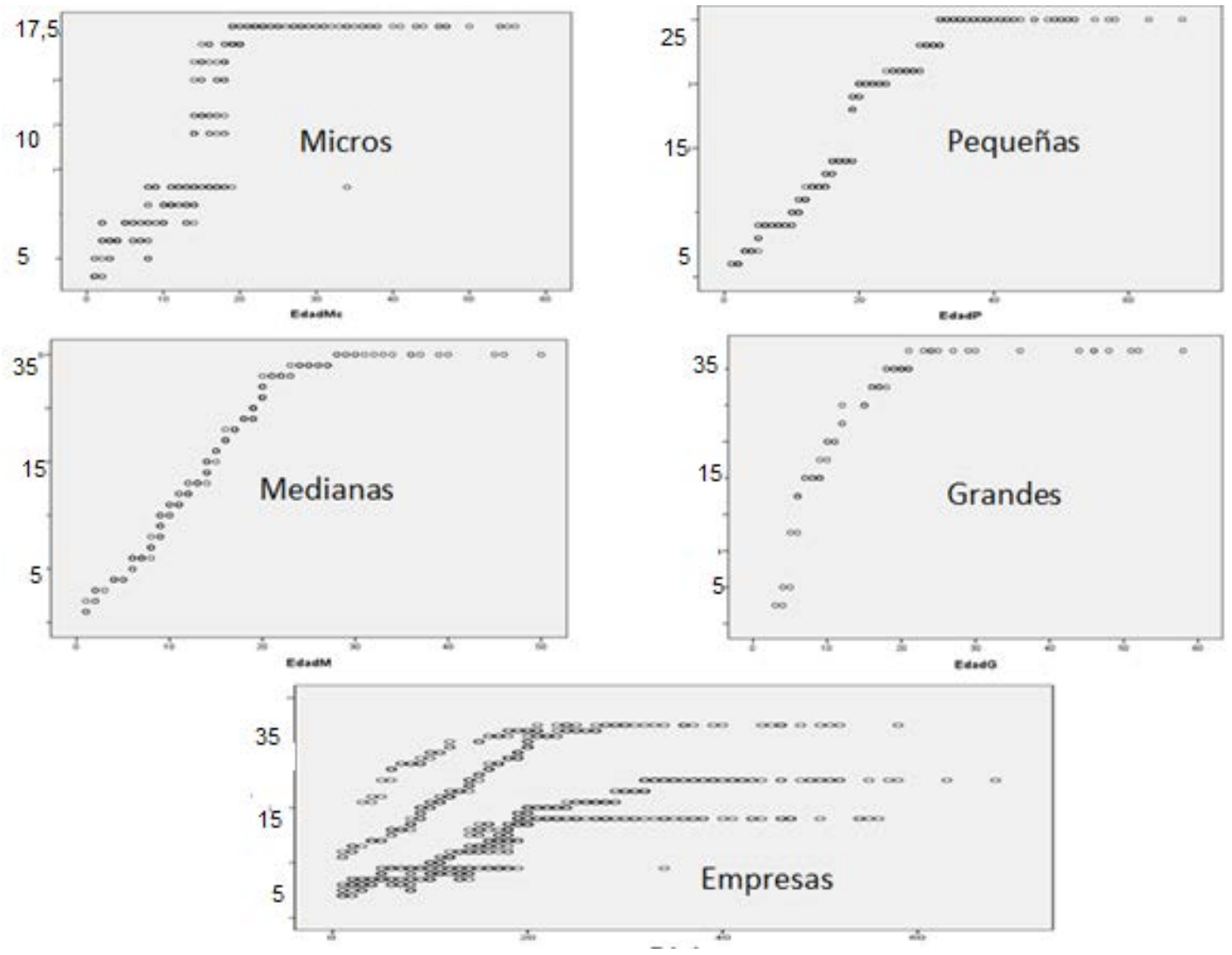

En la figura 4 se resume el nivel de desarrollo de cada una de las funciones de la $\mathrm{GRH}$ en los distintos tipos de empresas. En la medida en que se incrementa el tamaño de las empresas tiende a aumentar el nivel de desarrollo de las funciones. Las funciones menos desarrolladas en las micro y pequeñas empresas son la organización del trabajo y la evaluación del desempeño; mientras que la más aplicada es la de estimulación, que muestra comportamientos satisfactorios independientemente del tipo de organización. En general, la función más afectada es la de evaluación del desempeño, la cual no se aplica ni en las micro o pequeñas empresas, al menos no se reconoce como una actividad formalizada y objeto de planificación y control.

El comportamiento de la $\mathrm{GRH}$ en las empresas en su conjunto resulta inferior al de las empresas medianas y grandes; esto obedece a que en la muestra con que se trabajó existe un predominio de las micro y pequeñas empresas.

La función de organización del trabajo resulta poco desarrollada y no está formalizada. Prácticamente no existe división del trabajo o niveles de especialización, a no ser en determinadas empresas que se ocupan de productos como el desarrollo de software. Como parte de estas características, no disponen de la descripción 
de cargo o manuales de procedimientos de trabajo. Esta situación va mejorando en la medida que aumenta el tamaño de las empresas.

Figura 4. Nivel de aplicación de las funciones de la GRH por tipo de empresas

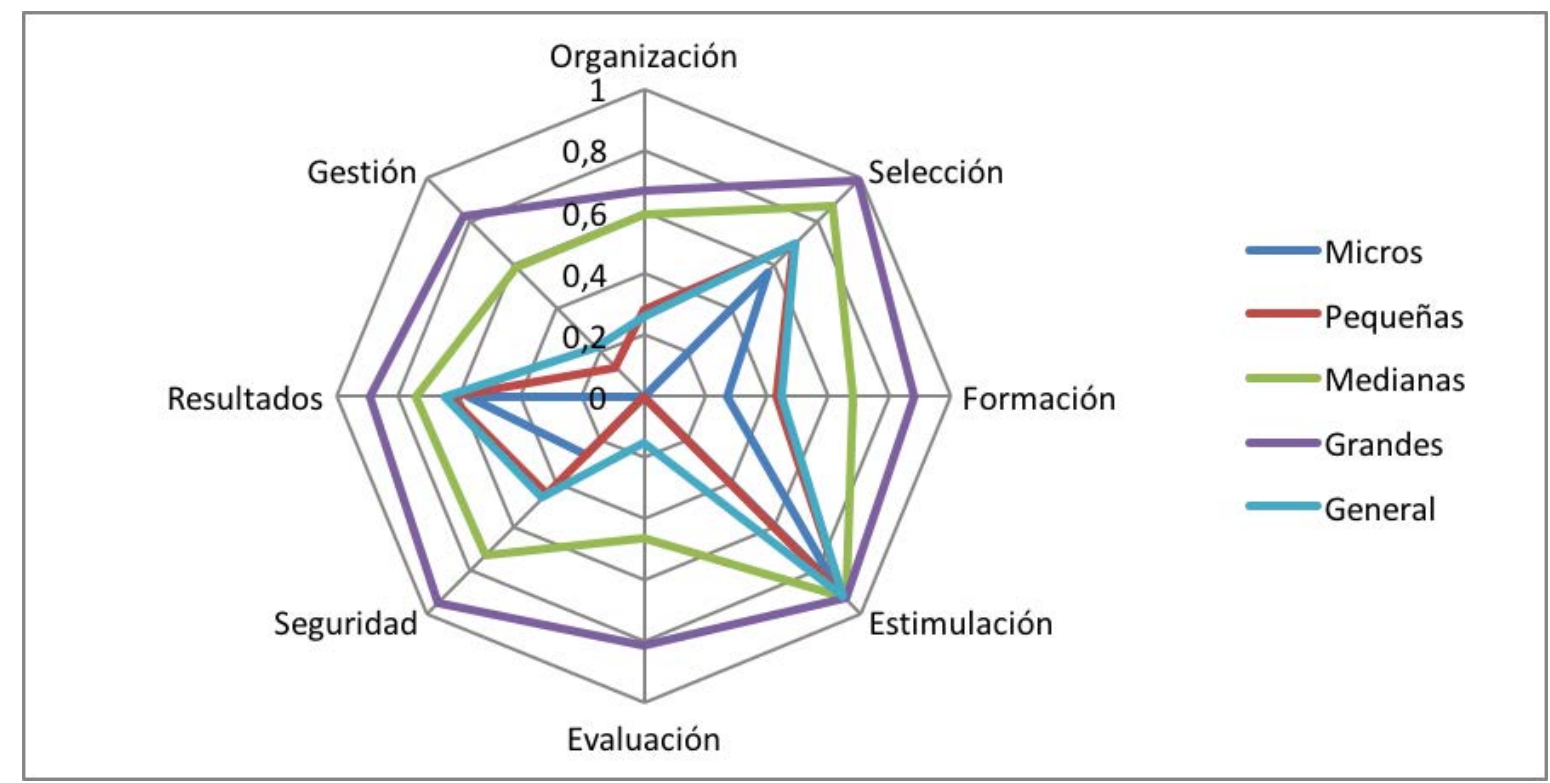

La selección del personal es una de las funciones más desarrolladas independientemente del tipo de organización. En la mayoría de ellas se hacen entrevistas de selección y se somete a los seleccionados a períodos de prueba. Por lo general, son seleccionados considerando requisitos físicos $y$, en menor medida, mentales; sin embargo, las pequeñas empresas no siempre consideran los requisitos mentales, o la aplicación de exámenes de selección para evaluarlos. De igual modo, en múltiples ocasiones no se toma en cuenta la certificación de los conocimientos generales o específicos.

La actividad de formación del personal tiende a mejorar con el tamaño de las empresas. Generalmente, las pequeñas y medianas empresas no planifican acciones de formación a partir de determinar las necesidades de aprendizaje, sino que esta se orienta a aspectos generales y no a la formación especializada, aunque sí se cumplen acciones de inducción y entrenamiento para la actividad.

La función de estimulación ${ }^{1}$ resulta ser la más favorecida en todas las empresas. Todas pagan salarios y un grupo de beneficios adicionales que corresponden al trabajo por ley; no obstante, la aplicación del pago por resultado tiende a ser menor en este tipo de organización. De forma general, se constató que la magnitud de la recompensa se incrementa en correspondencia con el tipo de empresa y esta oscila, como promedio al mes, entre 434.46 USD y 458.24 USD en las micro y pequeñas y entre 626.89 USD y 630.21 USD en las medianas y grandes.

En las mipymes, La función de evaluación del desempeño es nula; mientras que en las medianas si bien existe, no está formalizada o estandarizada, pero sí está relacionada con la descripción de los cargos. En las empresas grandes el problema fundamental se concentra en que no siempre está forma-

$1 \quad$ No se consideró como parte del análisis la aplicación de acciones de estimulación moral. 
lizada y no se aplican acciones de mejora en correspondencia con sus resultados.

Respecto a la función de seguridad, esta está establecida por ley, por lo que se manifiesta en mayor o menor medida en todas las organizaciones. En las pequeñas empresas, en algunos casos, solo se cumple a partir de la utilización de medios de protección o del análisis de accidentes si estos ocurren. En la medida en que el tamaño de la organización crece se elaboran inventarios de riesgos, planes de prevención y de mejora de las condiciones de trabajo.

En lo referente a los resultados que se persiguen en la GRH, las microempresas se concentran en lo fundamental, en el control del ausentismo $y$, en menor medida, en la permanencia, el compromiso y la satisfacción de los trabajadores. La búsqueda de estos indicadores tiende a mejorar dependiendo del tamaño de las empresas.

Como actividad de gestión, no existen evidencias de que estas se apliquen en las microempresas, aunque los directivos 0 propietarios de negocios asumen algunas de las funciones necesarias para garantizar los recursos humanos. Algunas pequeñas empresas asignan la función de GRH a un trabajador; sin embargo, esta no suele estar formalizada en manuales de gestión, no forma parte de un departamento independiente y no se concibe como parte de un proceso de mejora continua. En las empresas medianas, como promedio, la función la asume un departamento independiente, cuentan con procedimientos de trabajo y ejecutan acciones de mejora de la función. Esta situación tiende a mejorar aún más en las empresas grandes, aunque tanto la formalización de la actividad como las acciones de mejora de esta pueden ser incluso más representativas en ambos tipos de empresas.

En la figura 5 se muestra un resumen del comportamiento de las variables evaluadas en cada una de las funciones. Para veri- ficar si existían diferencias significativas en el desarrollo de la GRH por tipo de organización, se aplicó una prueba de comparación de medias para muestras independientes utilizando el estadígrafo de Kruskal-Wallis. Se puede garantizar que, con un nivel de confianza del $95 \%$, las diferencias en el nivel de desarrollo de la GRH por cada tipo de empresa son estadísticamente significativas. De igual modo, se comprobó que la diferencia entre el nivel de recompensas que ofertan las organizaciones, según el tipo de empresas, también resulta estadísticamente significativo.

\section{Discusión y conclusiones}

Los niveles de correlación encontrados entre el tamaño de las empresas y el grado de desarrollo de la actividad de GRH corresponden con los resultados expuestos por Krishnan y Hug (2017), con la particularidad de que en esta ocasión se contemplan los cuatro tipos de empresas, desde las microempresas hasta empresas con más de 200 trabajadores.

De igual modo, las funciones más desarrolladas por las micro y pequeñas empresas se relacionan con las acciones de recompensa y de selección del personal, aspecto ya corroborado en investigaciones precedentes (Cardon \& Stevens, 2004; Cellini \& Turner, 2019; Lozano-Reina \& SánchezMarín, 2020).

En estudios precedentes, los resultados relativos a las funciones de organización del trabajo (Aguinis \& Bakker, 2021; Han et al., 2020), formación (van Dijk et al., 2020) y evaluación del desempeño (Miao et al., 2020) no pudieron ser validados en el contexto de las micro y pequeñas empresas, pero sí se manifestaron en empresas medianas y grandes.

Independientemente del tamaño de la empresa se constató el desarrollo de la función de seguridad y salud en el trabajo, 
en correspondencia con los resultados de los estudios desarrollados por otros investigadores (Carpenter et al., 2016; McClellan \& Tekin, 2017), aunque se puso de manifiesto que la complejidad de la actividad aumenta con el incremento del tamaño de la empresa. Por último, aunque varía la frecuencia con que se establecen los objetivos de la
GRH en correspondencia con el tamaño de la organización, ya sea sobre retención del personal, ausentismo y productividad, u otros, los objetivos que se definen se relacionan con los propósitos analizados por otros investigadores (Borjas \& Doran, 2015; Hensvik \& Rosenqvist, 2019; Lohaus \& Habermann, 2019; Degbey et al., 2020).

Figura 5. Comportamientos de los ítems por dimensión
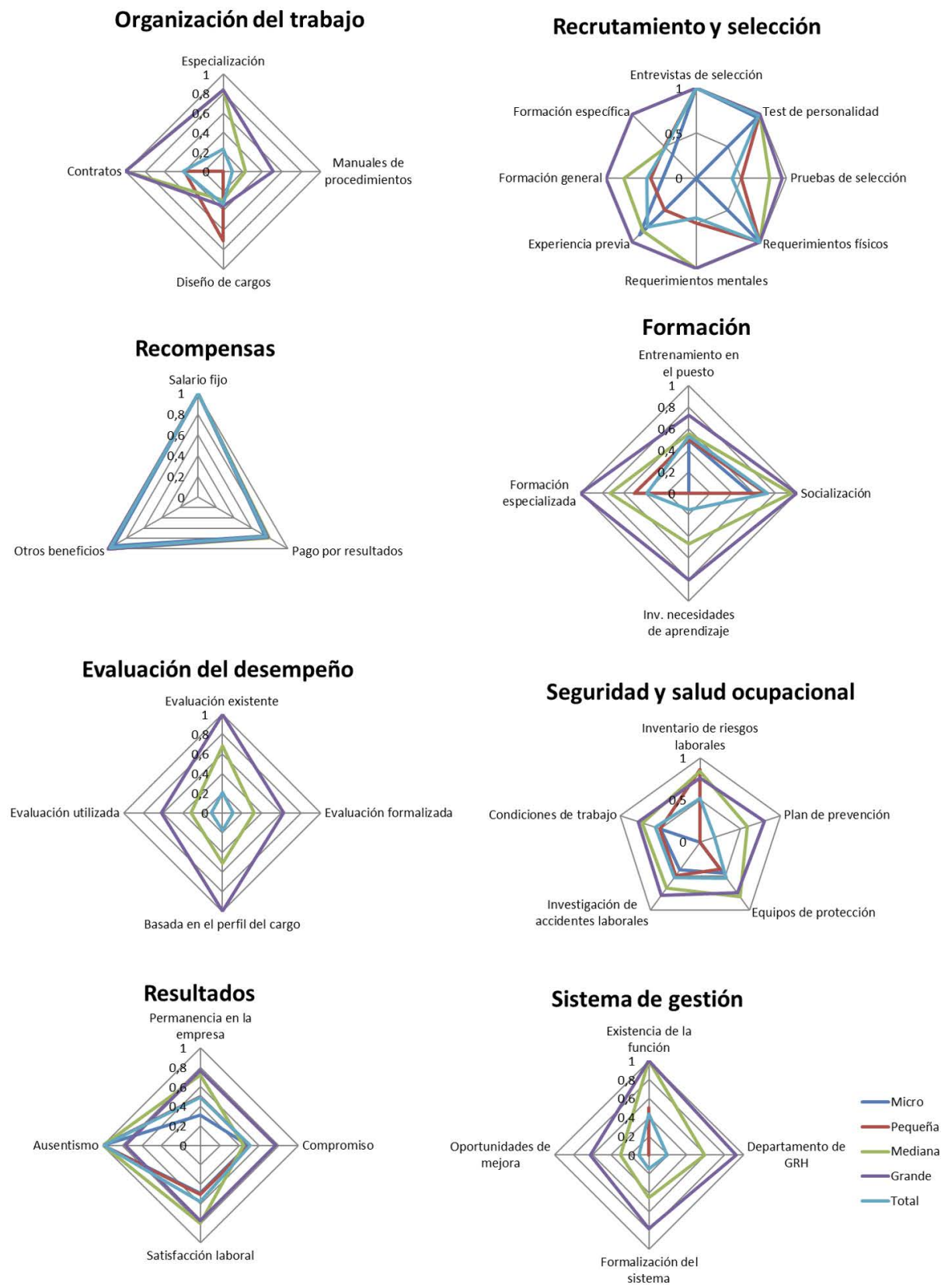
Los límites de las investigaciones se establecen a partir de la propia definición de la población objeto de estudio, cuyo comportamiento corresponde con las características socioeconómicas y legislativas donde operan las empresas, por lo que se recomienda evaluar si los resultados reportados se manifiestan de forma similar en otros contextos.

El acceso restringido a la información de algunas empresas impidió profundizar en otros aspectos relativos a la percepción de los trabajadores sobre la GRH, y evaluar el impacto de esta gestión en indicadores como el nivel de compromiso y satisfacción de los trabajadores respecto a la entidad donde laboran. Igualmente, no se evaluó la presencia de algunos rasgos propios de la GRH como su carácter estratégico, participativo y el enfoque por competencias, lo cual podría ser también objeto de análisis en investigaciones posteriores.

Como resultado de la investigación se pueden derivar algunas implicaciones gerenciales relacionadas con el reconocimiento de existencias de reservas para la mejora organizacional, asociadas a funciones de GRH, que todavía son susceptibles de mejora independientemente del tamaño de la organización. Otro aspecto que a criterio de los autores requiere de la implicación de los empresarios se asocia a la necesidad de reconocer que la formación gerencial para el caso de mipymes demanda adaptaciones, dado que no se debe asumir que los modelos de gestión que se desarrollan para empresas medianas y grandes son pertinentes para empresas de menor magnitud.

En conclusión, se refuerza el hecho de que la forma de concebir la GRH debe estar en correspondencia, en general, con las características de la empresa y, en particular, con su tamaño. Asimismo, se establece que es de esperar que la empresa, en la medida en que madure y se consolide, logre adoptar mejores prácticas de GRH. Todo lo anterior redunda en la idea de que no se manifiestan en igual me- dida, ni en idéntica forma cada una de las funciones que integran la GRH. Los resultados encontrados corresponden y se complementan con los resultados de investigaciones precedentes y se ajustan a las particularidades propias del entorno del objeto de estudio.

\section{Referencias}

Acikgoz, Y. (2019). Employee recruitment and job search: Towards a multi-level integration. Human Resource Management Review, 29(1), 1-13. https://doi.org/10.1016/j.hrmr. 2018.02.009

Aguinis, H., \& Bakker, R.M. (2021). Time is of the essence: Improving the conceptualization and measurement of time. Human Resource Management Review, 31(2), 100763. https:// doi.org/10.1016/j.hrmr.2020.100763

Ahammad, M.F., Glaister, K.W., \& Gomes, E. (2020). Strategic agility and human resource management. Human Resource Management Review, 30(1). https://doi. org/10.1016/j.hrmr.2019.100700

Barrett, R., \& Mayson, S. (2006). Exploring the intersection of HRM and entrepreneurship: Guest editors' introduction to the special edition on HRM and entrepreneurship. Human Resource Management Review, 16(4), 443-446. http:// dx.doi.org/10.1016/j.hrmr.2006.08.001

Batistič, S. (2018). Looking beyond - socialization tactics: The role of human resource systems in the socialization process. Human Resource Management Review, 28(2), 220-233. https://doi.org/10.1016/j. hrmr.2017.06.004

Borjas, G.J., \& Doran, K.B. (2015). Prizes and productivity: How winning the fields medal affects scientific output. Journal of Human Resources, 50(3), 728-758. https:// doi.org/10.3368/jhr.50.3.728

Bush, J.T. (2020). Win-Win-Lose? Sustainable HRM and the promotion of unsustainable employee outcomes. Human Resource Management Review, 30(3). https://doi. org/10.1016/j.hrmr.2018.11.004

Cardon, M.S., \& Stevens, C.E. (2004). Managing human resources in small organizations: 
What do we know? Human Resource Management Review, 14(3), 295-323. https:// doi.org/10.1016/j.hrmr.2004.06.001

Carpenter, C. S., Dobkin, C., \&Warman, C. (2016). The mechanisms of alcohol control. Journal of Human Resources, 51(2), 328-356. https:// doi.org/10.3368/jhr.51.2.0314-6240R

Cellini, S.R., \& Turner, N. (2019). Gainfully employed?: Assessing the employment and earnings of for-profit college students using administrative data. Journal of Human Resources, 54(2), 342-370. https://doi. org/10.3368/jhr.54.2.1016.8302R1

Combs, J. G., Jaskiewicz, P., Kristen, K., Shanine, K. K. \& Balkin, D. B. (2018). Making sense of HR in family firms: Antecedents, moderators, and outcomes. Human Resource Management Review, 28(1), 1-4. https:// doi.org/10.1016/j.hrmr.2017.05.001

Daspit, J.J, Kristen, M., Barnett, T., \& Long, R.G (2018). The emergence of bifurcation bias from unbalanced families: Examining HR practices in the family firm using circumplex theory. Human Resource Management Review, 28(1), 18-32. http://dx.doi. org/10.1016/j.hrmr.2017.05.003

Degbey, W.Y., Rodgers, P., Kromah, M.D., \& Weber, Y. (2020). The impact of psychological ownership on employee retention in mergers and acquisitions. Human Resource Management Review, 37(3) https://doi. org/10.1016/j.hrmr.2020.100745

Han, J., Sun, J.M., \&Wang, H.L. (2020). Do high performance work systems generate negative effects? How and when? Human Resource Management Review, 30(2). https:// doi.org/10.1016/j.hrmr.2019.100699

Hensvik, L., \& Rosenqvist, O. (2019). Keeping the production line running: Internal substitution and employee absence. Journal of Human Resources, 54(1), 200-224. https:// doi.org/10.3368/jhr.54.1.0516.7914r1

Instituto Nacional de Estadística y Censos (INEC). (2018, 30 de mayo). Visualizador de estadísticas productivas. https://bit. ly/2ZYkemx

Johnson, A.F., \& Roberto, K.J. (2019). Elections and selection: The role of political ideology in selection decisions. Human Resource Ma- nagement Review, 29(1), 14-27. https:// doi.org/10.1016/j.hrmr.2018.05.003

Krishnan, T.N., \& Hugh, S. (2017). Talent management and dynamic view of talent in small and medium enterprises. Human Resource Management Review, 27(3), 431-441. https://doi.org/10.1016/j.hrmr.2016.10.003

Lohaus, D., \& Habermann, W. (2019). Presenteeism: A review and research directions. Human Resource Management Review, 29(1), 43-58. https://doi.org/10.1016/j. hrmr.2018.02.010

Lozano-Reina, G., \& Sánchez-Marín, G. (2020). Say on pay and executive compensation: A systematic review and suggestions for developing the field. Human Resource Management Review, 30(2). https://doi. org/10.1016/j.hrmr.2019.01.004

McClellan, C., \& Tekin, E. (2017). Stand your ground laws, homicides, and injuries. Journal of Human Resources, 52(3), 621-653. https://doi.org/10.3368/jhr.52.3.06135723R2

Miao, C., Qian, S., Banks, G. C., \& Seers, A. (2020). Supervisor-subordinate guanxi: A meta-analytic review and future research agenda. Human Resource Management Review, 30(2). https://doi.org/10.1016/j. hrmr.2019.100702

Organización para la Cooperación y el Desarrollo Económico (OECD). (2015). Taxation of SMEs in OECD and G20 countries: OECD Tax Policy Studies, n. ${ }^{\circ} 23$. OECD Publishing.

Stone, C. \& Stone, D. L. (2015). Factors affecting hiring decisions about veterans. Human Resource Management Review, 25(1), 68-79. https://doi.org/10.1016/j. hrmr.2014.06.003

van Dijk, H., Shantz, A., \& Alfes, K. (2020). Welcome to the bright side: Why, how, and when overqualification enhances performance. Human Resource Management Review, 30(2). https://doi.org/10.1016/j. hrmr.2019.04.004

Wilkinson, A., Barry, M., \& Morrison, E. (2020). Toward an integration of research on employee voice. Human Resource Management Review, 30(1). https://doi. org/10.1016/j.hrmr.2018.12.001 